\title{
Impact of a formulation design on resultant dispersions of self-microemulsifying lipid systems
}

\author{
Naser M. Y. Hasan* \\ School of Pharmacy, Applied Science Private University, Amman, Jordan.
}

\begin{tabular}{l}
\hline ARTICLE INFO \\
\hline Received on: $18 / 08 / 2020$ \\
Accepted on: $26 / 11 / 2020$ \\
Available online: $05 / 03 / 2021$ \\
\\
\hline Key words: \\
SMEDDS, SEDDS, poorly \\
water-soluble drugs, \\
Ibuprofen liquigel capsule, \\
microemulsion.
\end{tabular}

\begin{abstract}
There is growing interest in the field of self-emulsifying lipid-based technology (SELBT) as an approach to enhance the bioavailability of poorly water-soluble drugs. As a result, the market has witnessed the introduction of more than 30 commercial products based on SELBT. In this investigation, key elements in the formulation design of oil systems which influence vehicle polarity and thus resultant dispersion profiles were optimized. Self-emulsification studies, particle size analysis, and phase behavior studies were carried out with and without drugs. Profiles were compared with commercial Ibuprofen liquigel capsules. Various factors including type of oil, cosurfactant, surfactant, cosolvent, and amount of loaded drug were found to influence dispersion profiles of the self-emulsifying lipid-based system. Furthermore, a significant correlation between total HLB of oil mix and mean emulsion droplet size of resultant aqueous dispersion was established. Also, emulsification of commercial Ibuprofen liquigel capsules has revealed drug precipitation, while self-microemulsifying lipid systems kept the drug in a dissolved state. In conclusion, despite the in vitro apparent failure of cosolvent-based formulation as in commercial Ibuprofen liquigel, these systems still have valid pharmacokinetic profiles.
\end{abstract}

\section{INTRODUCTION}

There is growing interest in the field of self-emulsifying lipid-based systems (SELBS) as an approach to improve the bioavailability of poorly water-soluble drug (Kim et al., 2019; Kovvasue et al., 2019; Sonia et al., 2019; Xiong et al., 2019). This interest is due to the fact that almost more than $40 \%$ of new APIs show low water solubility and thus poor oral bioavailability, high intra- and intersubject variability, and lack of dose proportionality (Sonia et al., 2019). Despite the fact that SELBS suit the most biopharmaceutical classification system category II compounds, which have low solubility and high permeability, other drug categories including class I, III, or IV can benefit from reformulation in oil vehicles. This is due to the fact that various constituents of SELBS, such as Cremophor EL, Tween-80, caprylic acid, citric acid, Labrasol, Myrj 52, Brij 30, polyethylene glycol (PEG) 400, and PEG 20,000, can enhance intestinal absorption,

\section{"Corresponding Author}

Naser M. Y. Hasan, School of Pharmacy, Applied Science Private University, Amman, Jordan.E-mail: N_hassan@asu.edu.jo initiate lymphatic absorption, or inhibit the catalysis of intestinal cytochrome P450 and the efflux of P-gp to drugs (Hasan et al., 2018; Samatha et al., 2014; Seo et al., 2013; Yan et al., 2012). SELBS for oral applications can be classified into self-emulsified or self-microemulsified drug delivery systems: self-emulsifying drug delivery system (SEDDS) or self-microemulsifying drug delivery systems (SMEDDS) based on oil droplet size of resultant dispersions. SEDDS are isotropic mixtures of oils, surfactants, and cosurfactants, which form under gentle agitation in aqueous media oil-in-water emulsion of oil droplet size $<5 \mu \mathrm{m}$. On the other hand, SMEDDS produce microemulsion dispersions of droplet size $<100$ $\mathrm{nm}$ due to the inclusion of high hydrophilic content in the lipid matrix. Since the advent of $\mathrm{Neoral}^{\circledR}$, an archetypical SMEDDS reformulation of earlier "Sandimmune" formulation, more than 30 products are now commercially available in the market, which are based on self-emulsifying lipid-based technology (Savla et al., 2017). Formulation design of successful SMEDDS requires lipid mixtures with optimum polarity to ensure fine aqueous dispersions with and without drugs, exhibit enough solubility to dissolve required dose and ability to hold the drug after dispersion, and thus prevent crystallization of drug and the flexibility to free the drug 
during digestion process to get good absorption profiles (Hasan, 2019a, 2014). In this study, various factors in the lipid mixture which affect dispersion profiles of SMEDDS with and without drug were investigated. This includes type of oil, cosurfactant, surfactant, cosolvent, and the amount of loaded drug. A correlation between total HLB of lipid mixture as an indicator of oil polarity and mean emulsion droplet size (MEDS) of resultant dispersions was established. Furthermore, a comparison was drawn between resultant dispersions of commercial Ibuprofen liquigel capsules which represent a hydrophilic cosolvent-based system and selfmicroemulsifying lipid formulation. This was in an attempt to withdraw the attention of the paradox of systems which may show apparent in vitro failure and yet have good bioavailability profiles.

\section{MATERIALS AND METHODS}

\section{Materials}

Captex 300 (caprylic/capric triglyceride), Capmul medium-chain mono- and diglycerides, Caprol PGE 860 (polyglycerol-10 mono-dioleate), and Acconon cc-6 (polyoxyethylene 6 caprylic/capric glycerides) were supplied as gifts by Abitec Corporation. Miglyol 812 medium-chain triglyceride (MCT) and Imwitor $988\left(\mathrm{C}_{8} / \mathrm{C}_{10}\right.$ mono/diglycerides) were supplied by Condea Chemie GmbH. Tagat TO [PEG-(25)glyceryl trioleate] was supplied by Goldschmidt AG, Germany. Cremophor EL (polyoxyethylene-(35)-castor oil), Cremophor RH40 (PEG-40 hydrogenated castor oil), Lutrol F127 (poloxamer 407), and Ibuprofen were obtained from BASF Corporation. Labrasol (caprylocaproyl polyoxyl-8 glycerides) and Maisine 35-1 (mixture of long-chain mono-, di-, and triglycerides) were obtained from Gattefosee. Soybean oil, a long-chain triglyceride (LCT), was purchased from Sigma-Aldrich. PEG 400 was obtained from Fluka. All water used was Mili Q water.

\section{Methods}

\section{Self-emulsification}

Self-emulsification experiments were carried out according to methods described by Hasan (2014). Excipients in the following order, oil, cosurfactant, surfactant, and cosolvent, were weighed in glass vials and then were hermetically sealed. Lipid mixtures were then heated in a thermostated water bath for 1 minute at around $50^{\circ} \mathrm{C}$ or at $80^{\circ} \mathrm{C}$ in the case of using solid materials such as Lutrol 127. Formulations were then vortexed and allowed to equilibrate at $25^{\circ} \mathrm{C}$ overnight in the oven. An amount of $1 \mathrm{~g}$ of each mixture was introduced into $100 \mathrm{ml}$ of Mili Q water in a 500 in a $500 \mathrm{ml}$ glass beaker. Emulsification was carried out in a $25^{\circ} \mathrm{C}$ thermostatically controlled mechanical shaker set at 100 oscillations per min for 15 minutes.

\section{Drug incorporation into lipid vehicle}

Ibuprofen at concentrations of either $100 \mathrm{mg}$ or 200 $\mathrm{mg} / \mathrm{g}$ lipid was added to glass vials containing SMEDDS. Each vial was sonicated and thoroughly vortexed.

\section{Particle size analysis}

Mean emulsion droplet diameter for lipid mixtures after dispersion was measured using light scattering techniques (QELS, Malvern model LO-C photon correlation spectrometer). Experiments were carried out in triplicate.
Table 1. HLB values for some of the excipients used in this investigation.

\begin{tabular}{lc}
\hline Excipient & HLB value \\
\hline Miglyol 812 & 1 \\
Imwitor 988 & 5 \\
Capmul MCM & 5 \\
Tagat TO & 11 \\
Cremophor EL & $12-14$ \\
Cremophor RH40 & $14-16$ \\
Labrasol & 14 \\
\hline
\end{tabular}

\section{Calculating HLB value of oil mixtures}

Total HLB value for lipid mix was calculated according to the following formula:

$\mathrm{HLBmix}=\left(\mathrm{HLB}_{1} \mathrm{XC}_{1}\right)+\left(\mathrm{HLB}_{2} \mathrm{X} \mathrm{C}_{2}\right)+\left(\mathrm{HLB}_{3} \mathrm{XC}_{3}\right) \ldots$, where $\mathrm{HLB}_{1}, \mathrm{HLB}_{2}, \mathrm{HLB}_{3} \ldots=\mathrm{HLB}$ values for each constituent in the oil mix; $C_{1}, C_{2}, C_{3} \ldots=$ proportion of each component in the oil mix $(\% w / w)$.

Table 1 shows HLB values for some of the excipients which were used in this investigation.

\section{Fate of drug after dispersion}

Ibuprofen liquigel capsules were opened and $1 \mathrm{~g}$ of their content equivalent to a $200 \mathrm{mg}$ drug was accurately weighed into a $500 \mathrm{ml}$ conical flask to which $100 \mathrm{ml}$ of distilled water was added. A volume of $1 \mathrm{ml}$ of formulation composed of Capmul mediumchain mono (MCM)/Labrasol/Cremophor RH40 (36/24/40) containing $200 \mathrm{mg}$ Ibuprofen was also transferred into a $500 \mathrm{ml}$ conical flask containing $100 \mathrm{ml}$ of distilled water. Dispersions were then emulsified as described in previous sections for 3 hours.

\section{Results and Discussion}

\section{Resultant dispersions of various lipidic systems}

Resultant dispersion of oil formulations aftermath emulsification event is of paramount importance, which influences bioavailability of lipid-based oral drug delivery systems. There are key factors within the lipid composition which affect characteristic profiles of aqueous dispersions of oil formulations. Among those factors are type of oil (source of triglycerides), whether it is long-chain triglycerides (LCTs) or medium-chain triglycerides (MCTs), the use of cosurfactant which aids in the mechanism of emulsification (Hasan, 2014), and the type of nonionic surfactant, whether it is hydrophilic (HLB $>12$ ) or hydrophobic $(\mathrm{HLB}<12)$. In comparison to LCTs, there is a consensus among researchers that MCTs $\left(C_{8}-C_{10}\right)$ are easily emulsified using various nonionic surfactants producing fine microemulsion systems. In general, when using LCTs, a higher concentration of surfactant is required to form microemulsions compared with MCTs (Patil et al., 2014). Nonetheless, subsequent self-microemulsifying formulations based on long-chain $\left(C_{18}\right)$ lipids of highly lipophilic drugs $\log p>$ 5 have significantly enhanced oral bioavailability when compared to medium-chain $\left(C_{8}-C_{10}\right)$ lipids (Hasan et al., 2015). On the other hand, MCT-based SMEDSS have nonetheless enhanced bioavailability in the case of the reformulation of lipophilic drugs of $\log \mathrm{P}$ between 2 and 5. The two physical characteristics of the resultant aqueous dispersions of oil formulations which have a profound impact on bioavailability are MEDS and sensitivity 
Table 2. Characteristics of resultant dispersions of various lipidic systems with varying degrees of hydrophilicity after emulsification with and without $100 \mathrm{mg} / \mathrm{g}$ Ibuprofen.

\begin{tabular}{|c|c|c|c|c|c|c|c|}
\hline \multirow{3}{*}{$\begin{array}{c}\text { No. } \\
1\end{array}$} & \multirow{3}{*}{$\begin{array}{l}\text { Formulation mix \% }(w / w) \\
(70 / 30)\end{array}$} & \multicolumn{2}{|c|}{ Presence of drug } & \multicolumn{4}{|c|}{ Type of resultant dispersion } \\
\hline & & \multirow{2}{*}{$\frac{\text { Without }}{\circ}$} & \multirow{2}{*}{$\begin{array}{c}\text { With } \\
\bullet\end{array}$} & \multicolumn{2}{|c|}{ SMEDDS } & \multicolumn{2}{|c|}{ SEDDS } \\
\hline & & & & & & $\circ$ & • \\
\hline 2 & Miglyol 812/Imwitor 988/Tagat TO (49/21/30) & $\circ$ & - & & & & \\
\hline 3 & Captex 300/Caprol PGE 860/Acconon cc-6 (10/30/60) & $\circ$ & $\bullet$ & & & & \\
\hline 4 & Captex 300/Caprol PGE 860/Acconon cc-6/Lutrol F127 (8/24/48/20) & $\circ$ & $\bullet$ & & & & \\
\hline 5 & Captex 300/Caprol PGE 860/Acconon cc-6/Lutrol F127 (6/18/36/40) & ○ & $\bullet$ & $\circ$ & $\bullet$ & & \\
\hline 6 & Miglyol 812/Imwitor 988/Cremophor EL (7/63/30) & $\circ$ & $\bullet$ & & & $\circ$ & $\bullet$ \\
\hline 7 & Miglyol 812/Imwitor 988/Cremophor EL/PEG (6.3/56.7/27/10) & $\circ$ & $\bullet$ & & & $\circ$ & $\bullet$ \\
\hline 8 & Miglyol 812/Imwitor 988/Cremophor EL/PEG (5.6/50.4/24/20) & $\circ$ & $\bullet$ & & & $\circ$ & $\bullet$ \\
\hline 9 & Miglyol 812/Imwitor 988/Cremophor EL (21/49/30) & $\circ$ & • & & & $\circ$ & $\bullet$ \\
\hline 10 & Miglyol 812/Imwitor 988/Cremophor EL/PEG (18.9/44.1/27/10) & $\circ$ & $\bullet$ & & & $\circ$ & $\bullet$ \\
\hline 11 & Miglyol 812/Imwitor 988/Cremophor EL/PEG (16.8/39.2/24/20) & $\circ$ & • & & & $\circ$ & $\bullet$ \\
\hline 12 & Miglyol 812/Imwitor 988/Cremophor EL (35/35/30) & $\circ$ & $\bullet$ & $\circ$ & $\bullet$ & & \\
\hline 13 & Miglyol 812/Imwitor 988/Cremophor EL/PEG (31.5/31.5/27/10) & $\circ$ & $\bullet$ & $\circ$ & $\bullet$ & & \\
\hline 14 & Miglyol 812/Imwitor 988/Cremophor EL/PEG (28/28/24/20) & $\circ$ & $\bullet$ & $\circ$ & $\bullet$ & & \\
\hline 15 & Capmul MCM/Labrasol/Cremophor RH40 (36/24/40) & $\circ$ & $\bullet$ & $\circ$ & $\bullet$ & & \\
\hline 16 & Captex 300/Capmul MCM/Cremophor RH40/ethanol (36/18/36/10) & $\circ$ & - & $\circ$ & $\bullet$ & & \\
\hline 17 & Soybean/Maisine 35-1/Cremophor RH40/ethanol (30/30/30/10) & $\circ$ & $\bullet$ & $\circ$ & • & & \\
\hline
\end{tabular}

SEDDS are considered to be the turbid systems of oil droplet size $>5 \mu \mathrm{m}$, while SMEDDS are dispersions of optical clarity of oil droplet size $<100 \mathrm{~nm}$.

of dispersions to the ionic strength of the emulsification media. Nano-sized emulsions have a relatively large interfacial area of contact with gut lumen fluids which mimic absorption through augmenting partitioning of drugs from the oil phase to the aqueous phase. Furthermore, it was found that ionic strength and electrolytes present in the emulsification media affect resultant dispersions of lipidic SMEDDS (Hasan, 2014, 2019a). This effect is minimized in the case of using a nonionic surfactant system with a relatively high HLB value $>12$.

Table 2 shows resultant dispersion characteristics after emulsification of various lipidic systems with varying degrees of hydrophilicity with and without $100 \mathrm{mg} / \mathrm{g}$ Ibuprofen. In this investigation, dispersions having a MEDS $<100 \mathrm{~nm}$ were classified as optically clear SMEDDS, while dispersions producing MEDS of $>100 \mathrm{~nm}$ were referred to as turbid SEDDS. In the case of a system composed of Miglyol 812/Tagat TO as in formulation no. 1, turbid emulsion was obtained with and without drugs. This system was thoroughly studied by Wakerly et al. (1986) and was found not to be influenced by the ionic strength of emulsifying media. Yet, by including three parts of Imwitor 988 (polar oil) in the Miglyol 812/Tagat TO binary mix as in formulation no. 2, clear SMEDDS aqueous dispersions were obtained without drug and turbid emulsions when drug was included in the oil preconcentrate. This system was found to be affected by the electrolytes present in the emulsification media (Hasan, 2014). Tagat TO in formulations no. 1 and 2 or Acconon cc- 6 in formulations no. 3-5 are considered nonionic lipophilic surfactants with low HLB values $<12$ and thus have shown very limited solubilization capacity of drugs aftermath event of emulsification. In the case of systems no. 3 , no. 4 , or no. 5 , SMEDDS dispersions were obtained without drugs. Nonetheless, only system no. 5 was able to produce SMEDDS with drugs. The system composed of Captex 300/Caprol PGE 860/Acconon cc-6 (no. 3) was thoroughly studied by Hasan (2020) and was found to be sensitive to the ionic strength of emulsification media and this effect was thwarted by including Lutrol F127 in the oil mix. Lutrol F127 (Poloxamer 407), as a hydrophilic cosolvent, has a high solubilization capacity of the drug. Hence, SMEDDS dispersions were produced in the presence of $100 \mathrm{mg}$ Ibuprofen/g lipid when $40 \% w / w$ of Lutrol F127 was included in the oil mix as in the case of system no. 5. Imwitor 988 as polar oil acts as a cosurfactant which was titrated in the oil mix at Miglyol 812/Imwitor 988 ratios of 5:5 (systems 12-14), 3:7 (systems 9-11), or 1:9 (systems 6-8). For systems composed of Miglyol 812/Imwitor 988/Cremophor EL, clear SMEDDS dispersions were obtained with and without drugs only when the ratio of Miglyol 812/Imwitor 988 in the lipid mix was 5:5 as in formulation numbers of 12-14. Cosurfactants such as Imwitor 988 or Capmul MCM are thought to stabilize the interface by penetrating into the void spaces among surfactant molecules in the surfactant film around the oil droplet and hence lowering the interfacial tension and increasing the interfacial fluidity (Constantinides and Scalart, 1997; Hasan, 2014; Nazzal and Khan, 2002). Optimum self-microemulsifying dispersions were obtained at Miglyol 812/Imwitor 988 ratios of 5:5, while other ratios produced turbid SEDDS with and without drugs albeit including PEG at $10 \%$ or $20 \% \mathrm{w} / \mathrm{w}$ in the lipid mix. In the case of systems containing Cremophor RH40, which is a well-known hydrophilic nonionic surfactant with high solubilization capacity, SMEDDS dispersions were obtained with and without drug as in formulation numbers of 13-15. The use of hydrophilic materials such as hydrophilic surfactants (HLB $>12$ ) and cosolvents is common in lipid-based self-emulsifying technology. The rationale behind their use is the production of very fine emulsions and increasing the solvent capacity of formulations containing drugs with a $\log \mathrm{P}$ between 2 and 4 (Pouton, 2000). Therefore, clear dispersions were obtained using medium-chain mono-, di-, and triglycerides as in Capmul MCM/Captex 300 (systems numbers 13 and 14) or long-chain glycerides as in soybean/Maisine 35-1 (system no. 15). 


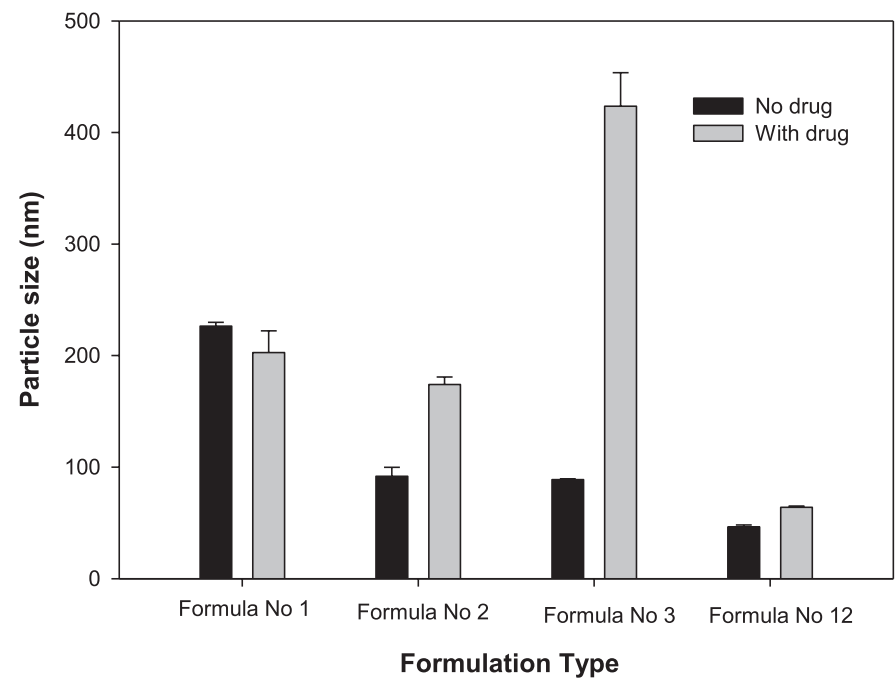

Figure 1. Particle size profiles of various lipid systems after dispersion; see Table 2 for oil composition.

Particle size profiles for resultant dispersions of various lipidic systems

There are key factors in the lipid formulations which affect particle size profiles of emulsions produced by lipid systems. Some of these elements include type of oil, the use of cosurfactants, type of nonionic surfactants, and the use of hydrophilic cosolvents. The more polar the lipid constituents in the mix are, the lower the MEDS of dispersions is obtained. This is true, as long as optimum oil : cosurfactant ratios and proper surfactant concentrations are used (Hasan, 2014, 2019b). Figure 1 shows particle size profiles of various lipid systems after dispersion. As for formulation no. 1, turbid SEDDS with MEDS of $>100 \mathrm{~nm}$ is obtained with and without drugs. This system is composed of highly lipophilic materials with a low HLB surfactant system (Tagat TO). Nonetheless, the inclusion of three parts of Imwitor 988 as a cosurfactant in formulation no. 2 has produced clear SMEDDS (oil droplet size $=91.8 \pm 8.1$ ) without drugs and turbid SEDDS (oil droplet size $=174.1 \pm 6.7$ ) with drugs. This provides clear evidence that the drug interferes in the mechanistic of emulsification of lipid-based systems. The same profile was shown in the case of system no. 3 which contains lipophilic constituents including Caprol PGE 860 as cosurfactant and Acconon cc-6 as a nonionic low HLB surfactant. Clear dispersion was obtained (MEDS $=32.8 \pm 1.3$ ) without drug and turbid SEDDS (MEDS $=423.6 \pm 30.1$ ) on including 100 $\mathrm{mg} / \mathrm{g}$ Ibuprofen. In the presence of drug, there is almost twofold increase in oil droplet size of formulation 3 which contains Acconon cc- 6 as a surfactant compared to formulation no. 2 which contains Tagat TO. This reflects the low solubilization capacity of Acconon cc-6 presumably due to its low polyoxyethylene content compared to Tagat TO. Nonetheless, as for formulation no. 12 which is considered a relatively high polar system, clear SMEDDS dispersions with and without drugs were obtained of oil droplet sizes of $46.3 \pm 1.9$ or $64.0 \pm 1.1$, respectively. Formulation 12 is composed of Cremohor EL, a hydrophilic nonionic surfactant of HLB between 12 and 14 and optimum oil : cosurfcatant ratio (Miglyol 812:Imwitor 988) of 5:5. The effect of the amount of drug

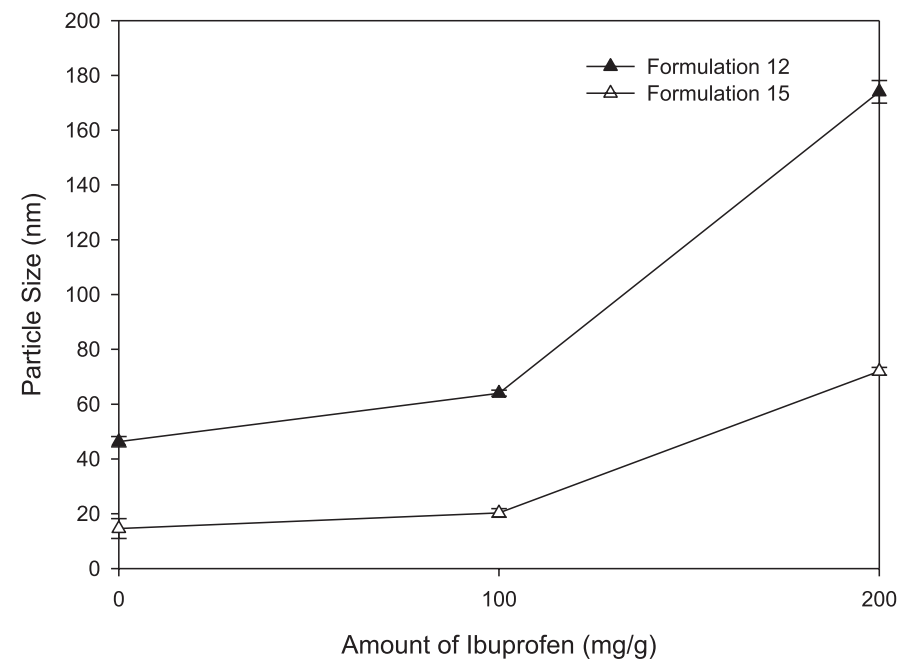

Figure 2. Effect of the amount of drugs on the MEDS profiles of resultant dispersions of various lipid vehicles with varying degrees of hydrophilicity.

loaded into lipid vehicles of varying degrees of hydrophilicity on the MEDS profiles of resultant dispersions is depicted in Figure 2. Formulations no. 12 and 15 are considered hydrophilic systems; yet, mix no. 15 has the highest polarity as it contains cosurfactant (Capmul MCM), cosolvent (Labrasol), and hydrophilic surfactant (Cremophor RH40) with no source of triglyceride. This has, however, reflected on the MEDS of resultant dispersions, as clear microemulsion dispersions were obtained in the case of system no. 15 at drug concentrations of 100 or $200 \mathrm{mg} / \mathrm{g}$. Yet, for system no. 12 clear SMEDS dispersions were obtained when the drug was only included at $100 \mathrm{mg} / \mathrm{g}$ but not $200 \mathrm{mg} / \mathrm{g}$. The effect of HLB value of oil mix on MEDS of resultant dispersion with and without drugs is shown in Figures 3 and 4. HLB value of oil mix correlates with the polarity of vehicle; the higher the HLB value of the formulation, the more hydrophilic the system. As Figure 3 depicts, there is a corresponding decrease in the oil droplet size with and without drug as the HLB value of the oil mix increases. Lipid self-microemulsifying mixtures containing high hydrophilic content will have high solubilization capacity of drug and hence have the ability to homogenize oil-in-water dispersed phases producing relatively small oil droplets. The relative difference in MEDS between dispersions with and without drug is widened at a relatively low HLB value. This difference, however, is abridged as the HLB value is gradually increased. Figure 3 can be simplified by plotting square root of HLB value for oil mix against ln MEDS as shown in Figure 4. Strong regression correlation $\left(R^{2}\right)$ of 0.96 was obtained in the case of dispersions without drug which could be utilized as a prediction tool describing the effect of HLB of oil mix on MEDS. Relatively lower $R^{2}$ was obtained (0.91) in the case of dispersions containing drug. This reflects the intervening effect of active substance on the mechanistic of emulsification. In another study, strong correlations were established between the HLB of lipid mixtures and the solubility of drugs in these vehicles (Devani et al., 2005).

The effect of PEG included in the SMEDDS preconcentrate and the amount of loaded drug on oil droplet size of resultant dispersion is shown in the contour plot depicted in Figure 5. In the case of lipid mixtures without loaded drug, 


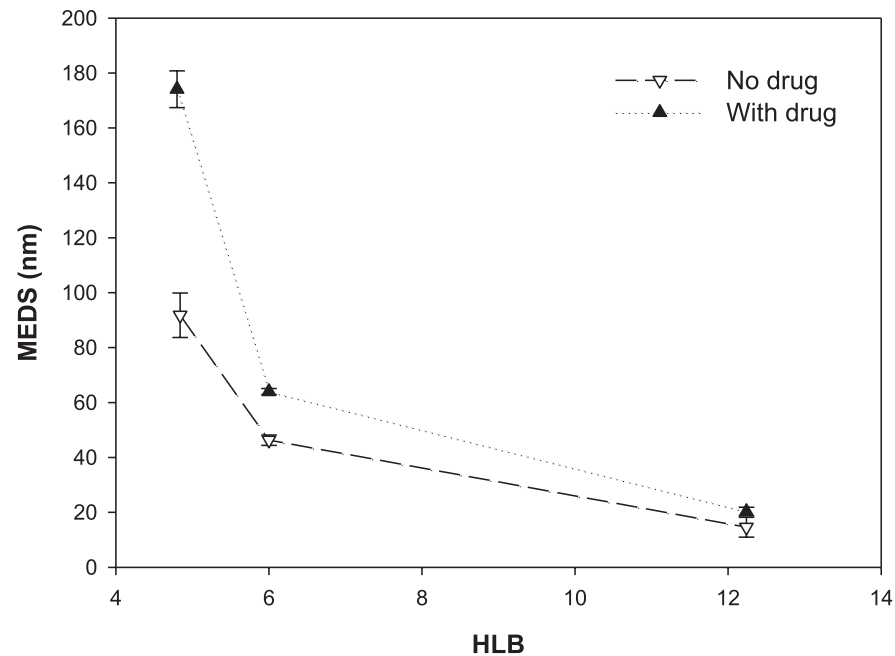

Figure 3. Effect of HLB values of oil mix on MEDS of resultant dispersion with and without drug.

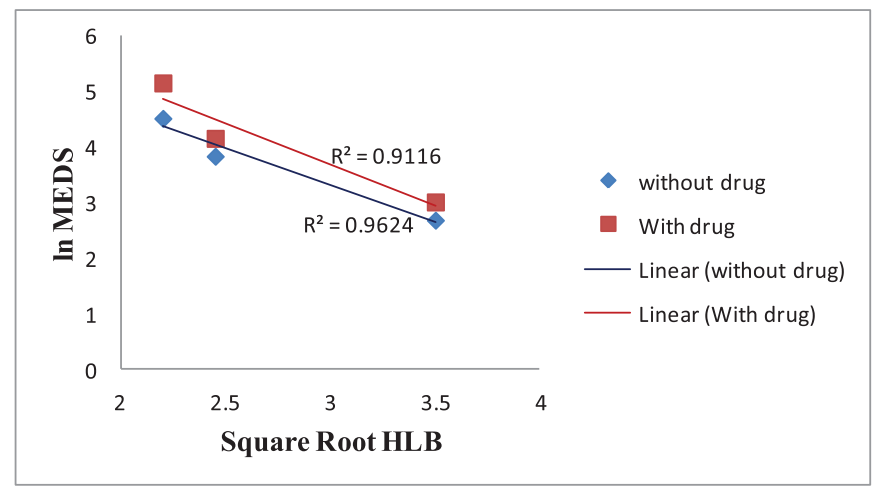

Figure 4. Correlation of the square root of HLB values of oil mix versus $\ln$ MEDS.

increasing concentrations of PEG in the lipid mixtures has minimal effect on MEDS of resultant dispersions. This, however, substantiates the idea that PEG does not interfere in the mechanistic of emulsification. At a constant amount of loaded drug, increasing PEG in the oil mixtures has ensued in a corresponding increase in MEDS. Despite the hydrophilic nature of PEG, it has shown low solubilization capacity compared to other hydrophilic cosolvents such as transcutol (Hasan, 2019a). Furthermore, at constant PEG concentrations, increasing the amount of loaded drug in the lipid vehicle results in progressive increases in MEDS. There is almost a fivefold increase in MEDS in the case of dispersions containing $200 \mathrm{mg} / \mathrm{g}$ drug compared to systems without any loaded drug.

It is generally accepted that medium-chain triglyceride (MCT) constituent in lipid-based systems emulsifies easily with high dynamic mobility at oil-water interface compared to LCT (Deckelbaum et al., 1990). It was found that the aqueous dispersion of SMEDDS containing LCT impedes the emulsification process by adding extra intermediate phases before forming clear $\mathrm{o} / \mathrm{W}$ microemulsion $\left(\mathrm{L}_{1}\right.$ phase) after sequential addition of water (Hasan et al., 2015). Furthermore, LCT-based SMEDDS has mimicked oral bioavailability of danazol vis-à-vis medium-chain $\left(C_{8}-C_{10}\right)$

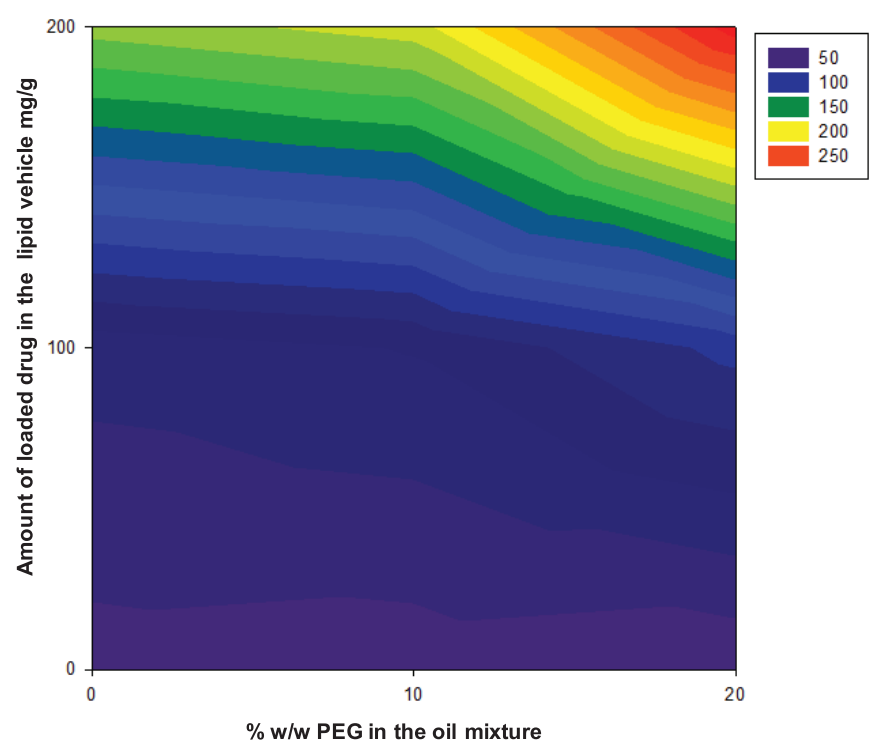

Figure 5. Contour plot of the effect of PEG included in the SMEDDS preconcentrate and the amount of the loaded drug on size of oil droplets of resultant dispersions.

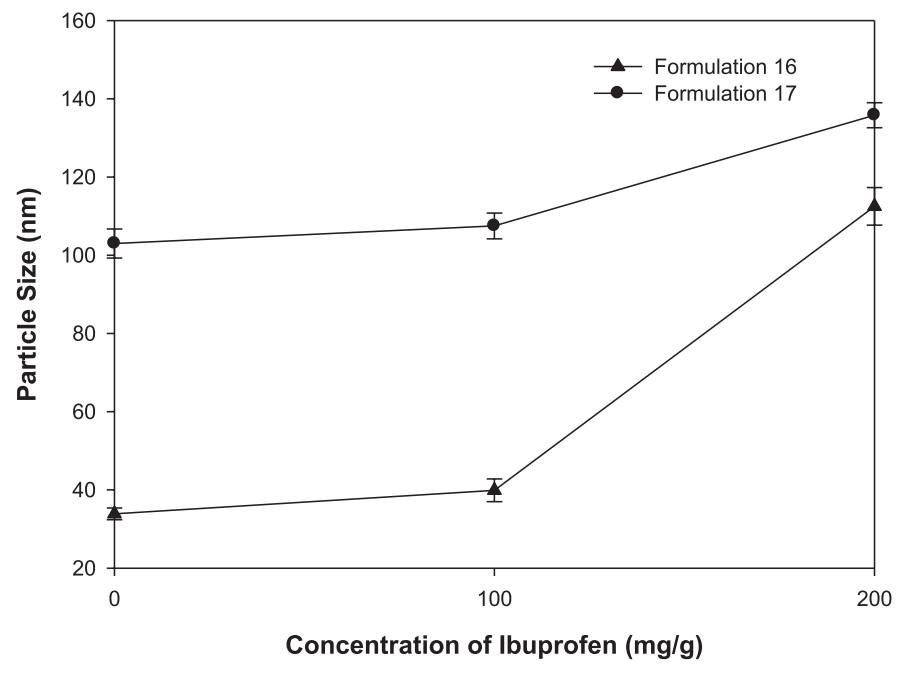

Figure 6. Effect of alkyl chain of source of triglyceride constituent in the lipid mix on the emulsification performance as measured by the oil droplet size at increasing drug concentrations.

lipid (MCT)-based SMEDDS. This enhancement in bioavailability is due to the association of LCT with the formation of lipoproteindependent (chylomicron) lymphatic transport path (Porter et al., 2004, 2007). The effect of the alkyl chain of the source of triglyceride constituent in the lipid mix on emulsification performance as measured by oil droplet size at increasing drug concentration is shown in Figure 6. Formulation no. 16 which contains Captex 300 as MCT has produced dispersions of particle size profiles which are relatively lower than formulation no. 17 which has soybean oil as LCT at all drug concentrations. This could be attributed to the fact that poorly water-soluble drugs with intermediate $\log \mathrm{P}$ values between 2 and 5 such as Ibuprofen (Subudhi and Mandal, 2013) or Nevirapine (Chudasama et al., 2014) have better solubilities 
(a)

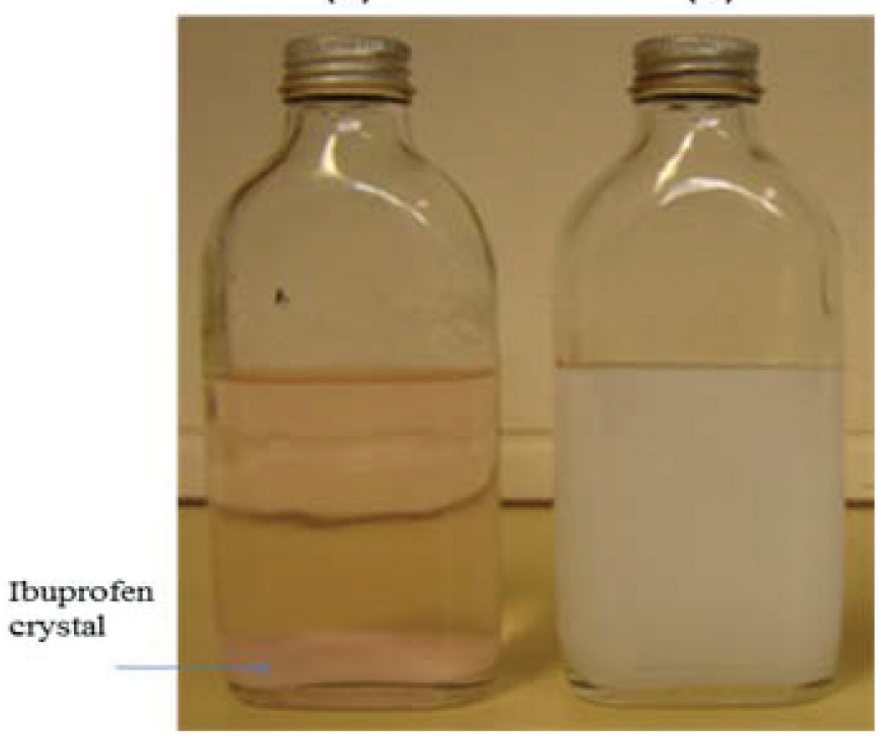

Figure 7. Emulsification profiles for commercial $200 \mathrm{mg}$ Ibuprofen liquid gel capsules (a) and self-microemulsifying lipid formulation containing $200 \mathrm{mg} / \mathrm{g}$ Ibuprofen (b).

in MCT than in LCT (Chudasama et al., 2014). This is due to the fact that MCTs are more polar oil than LCTs. MCT-based SMEDDS was shown to improve the bioavailability of Ibuprofen (Subudhi and Mandal, 2013) and Tipranavir (Aptivus ${ }^{\circledR}$, Boehringer Ingelheim Ltd.) (Sarpal et al., 2010).

\section{Solvent capacity of self-microemulsifying systems}

One crucial element for successful SMEDDS is the ability to retain the drug in a dissolved state during its passage in the lumen of gut. There are various factors that contribute to the loss of solvent capacity of lipid vehicle and thus may cause drug precipitation after dispersion of formulations. Among these are formulationbased factors that control hydrophilicity of vehicle including type and amount of oil (source of triglyceride and/or mixed glycerides), surfactant, and cosolvents. Endogenous-based factors include ionic strength of dispersion media and solubilization capacity of native surfactants such as bile salts and lecithin (Hasan, 2019a). The inclusion of increasing amounts of hydrophilic materials in the lipid mix may prompt drug crystallization in the lumen of gut. Hydrophilic components diffuse out from the vehicle after dispersion and thus any amount of drug carried by the hydrophilic constituents will be prone to precipitation. The propensity of drug precipitation during various stages of the dilution process which takes place during passage in the gut was investigated in various reports (Hasan et al., 2015; Hasan, 2019a; Mohsin and Alanazi, 2012; Mohsin et al., 2009; Porter et al., 2004). Despite the in vitro substantiated evidence of the propensity of the drug to come out of solution due to the hydrophilic nature of lipid vehicle, we have marketed products based on cosolvent systems such as Ibuprofen liquigel (Nurofen ${ }^{\circledR}$ by GSK or Advil ${ }^{\circledR}$ by Pfizer) (Al Lawati and Jamali, 2016) and free oil system as in Agenerase ${ }^{\circledR}$ (Rahman et al., 2012). These hydrophilic systems have shown reasonable bioavailability profiles albeit with the high possible inclination of drug crashing out during in vitro assessment studies. This paradox in the in vivo and in vitro correlation (IVIC) is due to the complex nature of the gastric content which allows very minimal drug crystallization to occur during digestion process, limited amount of free fluids in the gut, and the presence of endogenous surfactants and bile salts which facilitate solubilization and thus absorption process. The picture depicted in Figure 7 shows emulsification profiles for commercial $200 \mathrm{mg}$ Ibuprofen liquid gel capsules (a) and self-microemulsifying lipid formulation containing $200 \mathrm{mg} / \mathrm{g}$ Ibuprofen (b). Ibuprofen had crystallized out of solution after the dispersion of liquid gel capsules while SMEDDS maintained drug in solution. Hence, due to the balanced composition of the selfmicroemulsifying formulation which has enough amount of oil content, precipitation of drug after dispersion was circumvented. Nonetheless, in vivo studies have to be carried out to envisage implication of in vitro results on bioavailability profiles for both systems a and $\mathrm{b}$.

\section{CONCLUSION}

Factors which influence polarity of oil vehicle, including type of oil, cosurfactant, surfactant, and cosolvent were found to affect dispersion profiles of self-emulsifying lipid-based system. The direct correlation between total HLB of oil mix and MEDS of resultant aqueous dispersion with and without drugs was found. SMEDDS were found to keep the drug in solution after dispersion, while emulsification of commercial Ibuprofen liquigel capsules showed drug precipitation. This, however, should initiate further IVIC correlation studies

\section{ACKNOWLEDGMENTS}

The author acknowledges the Faculty of Pharmacy at Bath University for providing excellent facilities for research and for the lipid navigation team for carrying out some of the experiments, also Croda and BASF for sending samples as gifts, and finally the School of Pharmacy at Applied Science Private University for their support.

\section{AUTHOR CONTRIBUTIONS}

All authors made substantial contributions to conception and design, acquisition of data, or analysis and interpretation of data; took part in drafting the article or revising it critically for important intellectual content; agreed to submit to the current journal; gave final approval of the version to be published; and agree to be accountable for all aspects of the work. All the authors are eligible to be an author as per the international committee of medical journal editors (ICMJE) requirements/guidelines.

\section{FUNDING}

There is no funding to report.

\section{CONFLICTS OF INTEREST}

The authors report no financial or any other conflicts of interest in this work.

\section{ETHICAL APPROVALS}

This study does not involve experiments on animals or human subjects. 


\section{PUBLISHER'S NOTE}

This journal remains neutral with regard to jurisdictional claims in published institutional affiliation.

\section{REFERENCES}

Al Lawati H, Jamali F. Onset of action and efficacy of ibuprofen liquigel as compared to solid tablets: a systematic review and meta-analysis. J Pharm Pharm Sci, 2016; 19(3):301-11.

Chudasama AS, Patel VV, Nivsarkar M, Vasu KK, Shishoo CJ. In vivo evaluation of self emulsifying drug delivery system for oral delivery of nevirapine. Indian J Pharm Sci, 2014; 76(3):218-24.

Constantinides PP, Scalart JP. Formulation and physical characterization of water-in -oil microemulsions containing long-versus medium-chain glycerides. Int J Pharm, 1997; 158:57-68.

Deckelbaum RJ, Hamilton JA, Moser A, Bengtsson-Olivecrona G, Butbul E, Carpentier YA, Gutman A, Olivecrona T. Medium-chain versus long-chain triacylglycerol emulsion hydrolysis by lipoprotein lipase and hepatic lipase: implications for the mechanisms of lipase action. Biochem, 1990; 29(5):1136-42.

Devani MJ, Ashford M, Craig DQ. The development and characterisation of triglyceride based 'spontaneous' multiple emulsions. Int J Pharm, 2005; 300:76-88.

Hasan NMY. Effect of physicochemical properties of emulsions formed by self-emulsifying drug delivery systems (SEDDS) on the solubilization state of drug: in vitro study. Int J Appl Pharm, 2019a; 11(1):61-73.

Hasan NMY. Role of hydrophilic surfactants in the emulsification mechanistics of type III self-micro-emulsifying drug delivery systems (SMEDDS). Int J Appl Pharm, 2019b; 11(3):98-108.

Hasan NMY. Role of medium-chain fatty acids in the emulsification mechanistics of self-micro-emulsifying lipid formulations. J Saudi Pharm, 2014; 22:580-90.

Hasan NMY. Self-microemulsifying type II lipid class system: inhibiting salting out effect of electrolytes present in the emulsifying media. Int J Biol Pharm Allied Sci (IJBPAS) India, 2021

Hasan NMY, Hayajneh FM, Khaleel MA, Alharthi SA, Shahada HM, Almalki HF. Development of potential self-microemulsifying lipid formulation for the oral administration of curcumin. Int J Adv Pharm Biol Chem, 2015: 4(3):590-602.

Hasan NMY, Khaleel MA, Altwairqi AS, Alqurashi AG, Altwairqi AH. Effect of Self-micro emulsifying lipid formulation on the dissolution and compaction profiles of tablets containing theophylline; a BCS class I compound. J Appl Pharm Sci, 2018; 8(06):30-8.

Kim DS, Cho JH, Park JH, Kim JS, Song ES, Kwon J, Giri BR, Jin SG, Kim KS, Choi HG, Kim DW. Self-microemulsifying drug delivery system (SMEDDS) for improved oral delivery and photostability of methotrexate. Int J Nanomed, 2019; 14:4949-60.

Kovvasu SP, Kunamaneni P, Joshi R, Betageri GV. Selfemulsifying drug delivery systems and their marketed products: a review. Asian J Pharm, 2019; 13(2):73-84.

Mohsin K, Alanazi D. The fate of paclitaxel during in vitro dispersion testing of different lipid based formulations. J Drug Deliv Sci Technol, 2012; 22(2):197-204.

Mohsin K, Long MA, Pouton CW. Design of lipid based formulations for oral administration of poorly water-soluble drugs: precipitation of drug after dispersion of formulations in aqueous solution. $\mathrm{J}$ Pharm Sci, 2009; 98(10):3582-95.
Nazzal S, Khan MA. Response surface methodology for the optimization of ubiquinone self-nanoemulsified drug delivery system. AAPS PharmSciTech. 2002; 3(1):1-9.

Patil AS, Mahajan HD, Wagh RD, Deore BL, Mali BJ. Selfmicro-emulsifying drug delivery system (SMDDS): a novel approach for enhancement of bioavailability. Pharma Sci Monit, 2014; 5(1):133-43.

Porter CJ, Kaukonen AM, Boyd BJ, Edwards GA, Charman WN. Susceptibility to lipase-mediated digestion reduces the oral bioavailability of danazol after administration as a medium-chain lipid-based microemulsion formulation. Pharm Res, 2004; 21(8):1405-12.

Porter CJ, Trevaskis NL, Charman WN. Lipids and lipid-based formulations: optimizing the oral delivery of lipophilic drugs. Nat Rev Drug Discov, 2007; 6(3):231-48.

Pouton CW. Lipid formulations for oral administration of drugs: non-emulsifying, self-emulsifying and 'self-microemulsifying' drug delivery systems. Eur J Phram Sci, 2000; 11(2):93-8.

Rahman A, Hussain A, Hussain MS, Mirza MA, Iqbal Z. Role of excipients in successful development of self-emulsifying/microemulsifying drug delivery system (SEDDS/SMEDDS). Drug Dev Ind Pharm, 2012; 39(1):1-19.

Samatha A, Kumar GA, Reddy DS. Self-microemulsifying drug delivery systems: an attractive strategy for enhanced therapeutic profile. Int Sch Res Notices, 2014; 12(6):1-11.

Sarpal K, Pawar YB, Bansal AK. Self-emulsifying drug delivery systems: a strategy to improve oral bioavailability. Curr Res Inf Pharm Sci, 2010; 11(3):42-9.

Savla R, Browne J, Plassat V, Wasan KM, Wasan EK. Review and analysis of FDA approved drugs using lipid-based formulations. Drug Dev Ind Pharm, 2017;43(11):1743-58.

Seo YG, Kim DH, Ramasamy T, Kim JH, Marasini N, Oh YK, Kim DW, Kim JK, Yong CS, Kim JO, Choi HG. Development of docetaxelloaded solid selfnanoemulsifying drug delivery system (SNEDDS) for enhanced chemotherapeutic effect. Int J Pharm, 2013; 452(1-2):412-20.

Sonia A, Gupta R, Kumar PS. Formulation \& development of Self-Micro Emulsifying Drug Delivery System (SMEDDS) for oral bioavailability enhancement of a low soluble anti-diabetic drug: gliclazide. Adv Pharma Res, 2019; 6657176:01-15.

Subudhi BB, Mandal S. Self-microemulsifying drug delivery system: formulation and study intestinal permeability of ibuprofen in rats. J Pharm, 2013; 2013:328769.

Wakerly MG, Pouton CW, Meakin BJ, Morton FS. Selfemulsification of vegetable oil-nonionic surfactant mixtures: a proposed mechanism of action. ACS Symp Ser, 1986; 311:242-55.

Xiong Y, Zou Y, Chen L, Xu Y, Wang S. Development and in vivo evaluation of ziyuglycoside i-loaded self-microemulsifying formulation for activity of increasing leukocyte. AAPS PharmSciTech, 2019; 20:101-10.

Yan YD, Marasini N, Choi YK, Kim JO,Woo JS, Yong CS, Choi HG. Effect of dose and dosage interval on the oral bioavailability of docetaxel in combination with a curcumin self-emulsifying drug delivery system (SMEDDS). Eur J Drug Metab Pharmacokinet, 2012; 37(3):217-24

How to cite this article:

Hasan NMY. Impact of a formulation design on resultant dispersions of self-microemulsifying lipid systems. J Appl Pharm Sci, 2021; 11(03):100-106. 